\title{
Effect of Nanoclay and Silica on Mechanical and Morphological Properties of Jute Cellulose Polyethylene Biocomposites
}

\author{
Rezaur Rahman Md, ${ }^{1}$ Mizanur Rahman Md, ${ }^{2}$ Sinin Hamdan, ${ }^{2}$ Faruk Hossen Md, ${ }^{1}$ \\ Josephine Chang Hui Lai, ${ }^{1}$ Fui Kiew Liew ${ }^{2}$ \\ ${ }^{1}$ Department of Chemical Engineering and Energy Sustainability, Faculty of Engineering, University Malaysia \\ Sarawak, 94300 Kota Samarahan, Sarawak, Malaysia
}

${ }^{2}$ Department of Mechanical and Manufacturing Engineering, Faculty of Engineering, University Malaysia Sarawak, 94300 Kota Samarahan, Sarawak, Malaysia

\begin{abstract}
In this study, jute cellulose/polyethylene (PE) biocomposites were prepared using a hot press machine. Silica and nanoclay act as reinforcing agents in the composite system. The effects of clay and silica addition on the mechanical, thermal, and morphological properties of jute cellulose/PE biocomposites with different fiber loadings $(5,10,15$, and 20 wt $\%)$ were investigated. The biocomposites were characterized by Fourier transform infrared spectroscopy (FTIR), scanning electron microscopy, and thermogravimetric analysis. The mechanical properties were investigated using a universal testing machine. From FTIR results, it indicates that the $\mathbf{C}=\mathbf{0}$ stretching vibration had disappeared, while the intensity of peaks at 1718 and $1716 \mathrm{~cm}^{-1}$ appeared after addition of silica. The better interfacial bonding between jute cellulose/PE/silica biocomposites are reflected in the enhancement of the mechanical properties as well as thermal stability. The tensile strength and modulus had shown the highest values as well as higher activation energy for thermal decomposition. The surface area analysis result showed that the jute cellulose/PE/silica biocomposites have higher surface area and pore volume with less pore size. The manufactured biocomposites can be used in interior and exterior applications as well as a construction material. J. VINYL ADDIT. TECHNOL., 23:E113-E118, 2017. ( 2016 Society of Plastics Engineers
\end{abstract}

\section{INTRODUCTION}

Lignocellulosic materials are attracting the material engineering due to the development of composites materials. Lignocellulosic materials consisting of lignin, hemicellulose, and cellulose have turn out to be on alternative

Correspondence to: Md. M. Rahman; e-mail address: mizanurunimas@ gmail.com

Contract grant sponsor: Ministry of Higher Education Malaysia, University of Malaysia at Sarawak; contract grant number: ERGS/02 (08)/860/ 2912 (12).

DOI 10.1002/vnl.21554

Published online in Wiley Online Library (wileyonlinelibrary.com).

(C) 2016 Society of Plastics Engineers materials. Natural cellulose has been considered as the most potential substitute which is biodegradable, renewable, and biocompatible with polymer to fabricate the biomaterial [1]. It has attracted awareness of new materials for their low cost, light weight, and easy accessibility [2]. The average production of cellulose is $10^{11}-10^{12}$ tons per annum in nature. The consumption of cellulose in the world is $2 \times 10^{6}$ tons per annum [3]. The improvement of less environmental pollution and a large amount of energy can be saved by utilization of cellulose resource.

The advantages of the natural fiber reinforced composites are light weight, low cost, abundant availability, better thermal, and mechanical properties [4, 5]. Natural fibers, such as jute, flax, hemp, coir, sisal, wood, kenaf, cotton, and banana fibers are used in composites preparation [6]. In addition, jute fiber is the most inexpensive than other natural fibers. Jute fiber is well-known to have outstanding tensile strength and a high modulus [7]. Jute is a natural biodegradable renewable lignocellulose materials composed generally of cellulose (58-63\%), hemicellulose (20-22\%), lignin (13-15\%), pectin, and trace masses of organic and inorganic pigments [8]. Cellulose is the highest component of jute fiber, which is resistant to alkali but is hydrolyzed in acid [9]. The cellulose structure of the fibers is well-known through crystalline and amorphous regions, whereas hemicellulose and lignin are completely amorphous. Cellulose crystalline region contains strong hydroxyl groups [10]. The amorphous hemicellulose and lignin are totally removed by acid and alkali treatments [11-13]. Cellulose surfaces reduce moisture absorption, improved the thermal, mechanical properties as well as improve the wettability of cellulose and polyethylene (PE) matrix composites [9, 14]. PE is the most suitable matrix because it has excellent properties such as low density, great softening point, good flex life, good surface hardness, and excellent electrical properties [15]. Due to the aforementioned reasons, jute and PE are unique materials for preparation of biocomposites. 